\title{
Modelo sustentable de gestión editorial en Acceso Abierto en instituciones académicas. Principios y procedimientos
}

Sustainable model of editorial management in Open Access in academic institutions. Principles and procedures

\section{Guillermo Banzato}

Universidad Nacional de La Plata-CONICET, Argentina

gbanzato@fahce.unlp.edu.ar

Cecilia Rozemblum

Universidad Nacional de La Plata, Argentina

ceciroz@fahce.unlp.edu.ar

\section{ReSUMEN:}

El objetivo de este trabajo es desarrollar los principios fundacionales que dan sustento teórico y político a la gestión de revistas científicas en la Facultad de Humanidades y Ciencias de la Educación de la Universidad Nacional de La Plata, mostrar un flujo completo de procesamiento, edición, publicación e inclusión de artículos de revistas en bases de datos y en el repositorio institucional, demostrando la practicidad de la tecnología RedALyC-AmeliCA y mostrar sus costos básicos.

Palabras Clave: Soberanía del conocimiento, Acceso Abierto, Edición científica, Evaluación científica.

\section{Abstract:}

The objective of this work is to develop the foundational principles that give theoretical and political support to the management of scientific journals in the Faculty of Humanities and Education Sciences of the National University of La Plata, show a complete flow of processing, editing, publication and inclusion of journal articles in databases and in the institutional repository, demonstrating the practicality of the RedALyC-AmeliCA technology and showing its basic costs.

KEYwORDS: Sovereignty of knowledge, Open Access, Scientific edition, Scientific evaluation.

\section{INTRODUCCIÓN ${ }^{1}$}

En los últimos años de uso intensivo de las tecnologías, nos encontramos en un escenario que considera a la información como commodity, como mercancía de uso comercial, como bien producido masivamente cuyo valor está en la relación oferta-demanda, sin diferenciación en función de quién o dónde se produce.

Para los que formamos parte de la comunidad de actores de la producción de información científica, salir de esta presión es un desafío que requiere convertirnos en desarrolladores de capacidades diversificadas que no sean transformables en commodities. Esta estrategia se basa en incidir en la cadena de valor con mayor calidad.

(Bosch, 2013)

Este lúcido programa de trabajo institucional que desarrolló Mela Bosch durante su gestión en la dirección del CAICYT es un verdadero incentivo para quienes investigamos y gestionamos en edición científica. Asimismo, el movimiento de Acceso Abierto ha ido detallando las condiciones que debe cumplir una publicación científica para estar al alcance de todo el público, a partir de las conocidas Declaraciones denominadas $3 \mathrm{~B},{ }^{2}$ aunque recientemente se ha comenzado a debatir más específicamente acerca de cuán abierto es el Acceso Abierto si las principales economías del mundo se pronuncian a favor de cargar al autor los costos de edición, tal como se propone en el Plan S (Becerril-García, 2019), por el contrario, en el Sur Global se está proponiendo una base de datos inclusiva ${ }^{3}$ y una consideración más amplia de los sistemas 
de evaluación, ${ }^{4}$ al tiempo que se ha aportado a la consideración del conocimiento como un bien común (Aguado-López y Vargas Arbeláez, 2016; Vargas Arbeláez, 2014).

Estos debates implican considerar cómo llevar adelante estas propuestas, de qué manera organizar los flujos de trabajo y cómo calcular los costos para las instituciones de ciencia que quieran asumir el compromiso de gestionar sus revistas científicas. Nicolás Caitán realizó un análisis de la relación entre el aumento de los costos de suscripción y la aparición de propuestas en Acceso Abierto, abogando por políticas públicas que fomenten estos esfuerzos (Caitán, 2011), si bien no prestó debida atención a que las universidades, y no solamente las sociedades académicas, estaban produciendo revistas por la vía dorada, vinculadas estrechamente a repositorios.

El tema que nos ocupa ha sido muy poco abordado, pese a la constante disputa Acceso Abierto vs. edición comercial. Aunque la decisión acerca de publicar en uno u otro modelo no pasa exclusivamente por una cuestión económica, no conocemos trabajos que permitan hacer un análisis detallado de cuáles son los costos, al menos en América Latina. En una primera aproximación al tema en Rozemblum, Bava, Unzurrunzaga y Banzato (2014), ocasión en la que estábamos preocupados por los costos y beneficios de indizar en las principales bases de datos latinoamericanas, concluimos que nuestro modelo, en el que la edición está sostenida por profesionales bibliotecarios utilizando el gestor de contenidos Open Journal System (OJS), agiliza y optimiza la indización, los costos son menores que los de imprimir y los beneficios mayores. Pero claro está nunca editar e indizar es gratis, determinamos en ese momento que el procesamiento de 116 artículos de cuatro revistas para incluirlos en las bases de datos SciELO, RedALyC y DOAJ insumía 171,5 h a un costo de $\$ 8.331,7$, el $97 \%$ de este se destinaba a SciELO. ${ }^{5}$ En ese sentido, extrapolamos nuestros cálculos a la totalidad de revistas del Núcleo Básico de Revistas Científicas Argentinas (en ese año 109), determinando que ese procesamiento le habría costado al Estado el 0,1\% de lo que invertía la Biblioteca de Ciencia y Técnica del entonces Ministerio de Ciencia y Técnica para comprar el acceso a revistas de editoriales comerciales.

Utilizando los datos de la base de WoS desde 1973 hasta 2013, Larivière, Haustein y Mongeon (2015) determinaron que las cinco empresas más poderosas del mercado editorial de Occidente (Elsevier, Taylor \& Francis, Wiley-Blackwell, Springer y Sage Publications) tienen más artículos que todas las otras empresas más pequeñas, pero en promedio tienen menor impacto científico. El crecimiento de la participación de estas empresas en el mercado, según los autores, se debe a la creación de nuevas revistas y a la compra de revistas a sus competidoras más débiles. Argumentan que en la era digital estas empresas se beneficiaron aumentando su parte en el total de las publicaciones y haciendo cada vez más dependientes a las comunidades científicas. Al mismo tiempo, aumentaron sus ganancias en porcentajes comparables a las empresas más rentables de otras ramas de la economía como bancos, automotrices y medicamentos. Lo más interesante es que para estos autores

los costos fijos o de primera copia comprenden la preparación, selección y revisión de manuscritos, así como la edición y el diseño de copias, la redacción de editoriales, la comercialización, los salarios y el alquiler, los dos más importantes de los cuales, la redacción y revisión de manuscritos son proporcionados de forma gratuita por la comunidad académica. ${ }^{6}$

Asimismo, con la era digital los costos variables compuestos por imprenta y distribución devinieron en marginales, lo que los lleva a preguntarse por qué el colectivo académico sigue aplicando tanto presupuesto en las empresas editoras (Larivière, Haustein y Mongeon, 2015).

Claudio-González, Martín-Barranera y Planas nos ofrecen una mirada basada en una encuesta que abarca 561 revistas, sobre las 1.280 que estaban incluidas en la base de datos Dulcinea ${ }^{7}$ en 2012 (actualmente son 1.790). En cuanto a la estructura de ingresos la mayoría recibe subsidios de la administración pública, especialmente las universitarias; entre los gastos se destacan los servicios externos (no indican cómo se componen), personal y bienes corrientes, mientras que los costos están principalmente concentrados en la producción (maquetación, imprenta). Con respecto a la estructura organizativa abundan los editores que tienen que hacer las veces de administrador, con un personal mayoritariamente voluntario y con exigua 
dedicación horaria. Como los autores no cruzan variables no podemos saber los datos exclusivos de las revistas únicamente digitales (Claudio-González, Martín-Barranera y Planas, 2017).

En la Facultad de Humanidades y Ciencias de la Educación (FaHCE) de la Universidad Nacional de La Plata (Argentina) veníamos trabajando desde 2005 con la tecnología SciELO para poder incluir las revistas que fueron siendo invitadas a este portal, pero sin beneficios para la propia edición de las revistas, excepto la enorme visibilidad que nos daba ese portal. Hacia finales de 2012, con la instalación de varias revistas en el portal OJS de la FaHCE el flujo de edición no incluía el marcado que requería SciELO para la publicación, sino que se hacía luego de la publicación, sólo con fines de visibilidad, ya que es un sistema muy engorroso y lento para trabajar. Además, en 2014, SciELO comienza a tomar decisiones que no cumplían con nuestras expectativas del Acceso Abierto (Aguado López, 2018; Repiso, 2014), por lo cual hemos cambiado las prioridades de nuestro flujo de trabajo, redireccionando nuestro esfuerzo al sistema de marcado XML-JATS de RedALyC. De todas maneras, se está trabajando en la articulación de los sistemas adaptando los archivos de salida de RedALyC para enviarlos a SciELO y así optimizar el trabajo -hacerlo una sola vez- y no perder la gran visibilidad de SciELO.

El objetivo de este trabajo es desarrollar los principios fundacionales que dan sustento teórico y político a nuestra gestión, mostrar un flujo completo de procesamiento, edición, publicación e inclusión de artículos de revistas en bases de datos y en el repositorio institucional, demostrando la practicidad de la tecnología RedALyC-AmeliCA y mostrar sus costos básicos.

\section{Los PRINCIPIOS DE LA EDICIÓN CIENTífica en ACCeso Abierto}

Según nuestra experiencia, la sustentabilidad de un modelo de gestión en edición científica en Latinoamérica se basa en instituciones de ciencia sostenidas por fondos públicos; en la actividad vocacional del colectivo de editores, autores y revisores que hacen su trabajo como parte de su desarrollo profesional, sin remuneración adicional; en el desarrollo de revistas en Acceso Abierto, que no cobran por procesamiento de artículos (APC, en inglés); en la defensa de los derechos a partir de la adopción de Licencias Creative Commons Atribución - No comercial - Compartir Igual (BY-NC-SA por sus siglas en inglés), tal como lo postula la Declaración de México (LATINDEX-REDALYC-CLACSO-IBICT, 2017); en la edición exclusivamente electrónica abandonando el formato papel; en la coordinación técnica sustentada por la propia institución.

Para consolidar la sustentabilidad hemos desarrollado un modelo de gestión tripartito para profesionalizar la edición científica en el que interactúan las autoridades institucionales, sosteniendo políticamente los principios del Acceso Abierto y disponiendo los recursos humanos y económicos; los editores científicos con su red de autores y evaluadores para conseguir los mejores artículos posibles; los gestores de contenidos que forman un equipo interdisciplinario en el que confluyen diseñadores, correctores de estilo, bibliotecarios e informáticos para editar los trabajos, llevar adelante la plataforma de gestión y realizar las tareas de visibilidad en bases de datos y repositorios (Rozemblum y Banzato, 2012).

Asimismo, este modelo se basa en el conocimiento como bien común, de modo tal que, así como para la gobernanza de aquellos bienes comunes relacionados con la naturaleza y la producción agraria se ha apelado a la soberanía alimentaria, ${ }^{8}$ postulamos una soberanía del conocimiento para el manejo de los saberes ancestrales y académicos, definida como

la potestad de producir, conocer, enseñar, compartir y preservar los saberes ancestrales y los científicos, propios y globales, en Acceso Abierto.

La facultad de generar las herramientas para que esos conocimientos estén alojados en servidores territorialmente localizados en las comunidades que los generan y que los sistemas de conservación digital también estén territorialmente situados. El derecho a desarrollar sistemas de gestión y evaluación del conocimiento científico, sostenidos por fondos públicos, que tengan en cuenta las condiciones locales y regionales de productividad y sostenibilidad, las relaciones interinstitucionales, las formas propias de generación y diseminación de los saberes (Banzato, 2018). 


\section{LOS PROCEDIMIENTOS}

Sin dudas, las revistas latinoamericanas cuentan con calidad científica, toda vez que los editores han mantenido una selección de trabajos originales y han sido consecuentes en la constante revisión por pares. Sin embargo, todavía hay serias dificultades para que la mayoría de ellas consiga ser indexada en una base de datos, por lo que se necesita dar un salto exponencial en calidad editorial para sumar valor agregado. Para mejorar este aspecto las características deseables del modelo sustentable de gestión editorial son: maquetación semántica que identifique metadatos, proceso que debe realizarse una sola vez; interoperabilidad para permitir la cosecha múltiple lo más automáticamente posible; visibilidad procurando la mayor cantidad de puntos de acceso a la red; identificadores persistentes (DOI, Handle, u otras formas de trabajo con URIs); indicadores de evaluación diversos, tal como lo recomienda la Declaración de San Francisco (DORA, 2012).

El sistema de marcado Marcalyc/xml replicado actualmente en AmeliCA/xml ${ }^{9}$ nos ayuda a aportar valor agregado a nuestros contenidos. En este modelo de gestión los actores involucrados efectúan procesos con herramientas específicas, tal como se indica en la figura 1.

FIGURA 1

Modelo de gestión: actores, procesos y herramientas

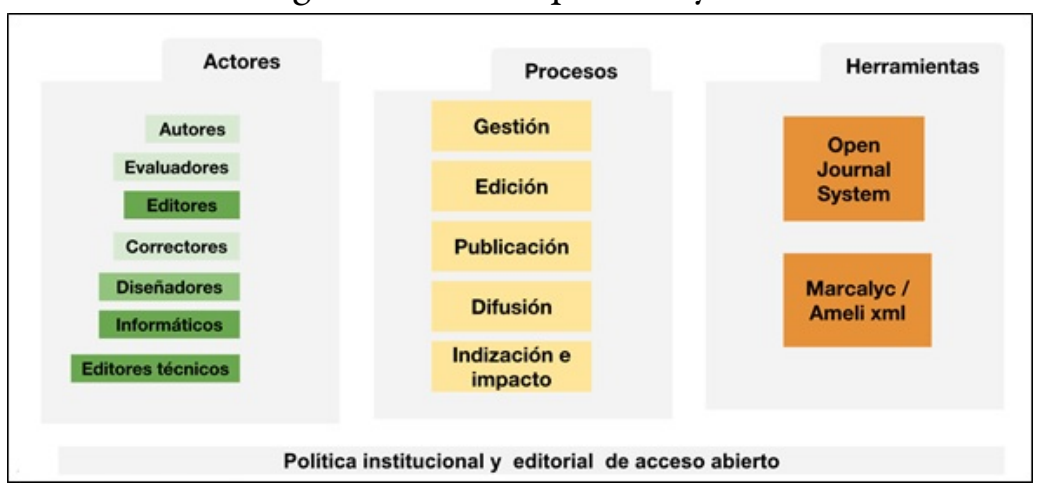

Elaboración propia

Tal como se observa -y se comentó anteriormente-, el modelo se sostiene por una política institucional constante que lo apoya política e ideológicamente, como así también en el Acceso Abierto.

Este modelo se desarrolla con el flujo de trabajo que se puede ver en la figura 2. En este se distinguen 3 colores: celeste, donde el actor es el editor o autor; el verde, cuyos actores son los profesionales del equipo técnico de edición (bibliotecarios, informáticos, diseñadores); y el amarillo, que muestra que la tarea de marcado, en cualquiera de los sistemas, la realizan bibliotecarios externos al equipo técnico. 
FIGURA 2

Flujo de trabajo del modelo de gestión

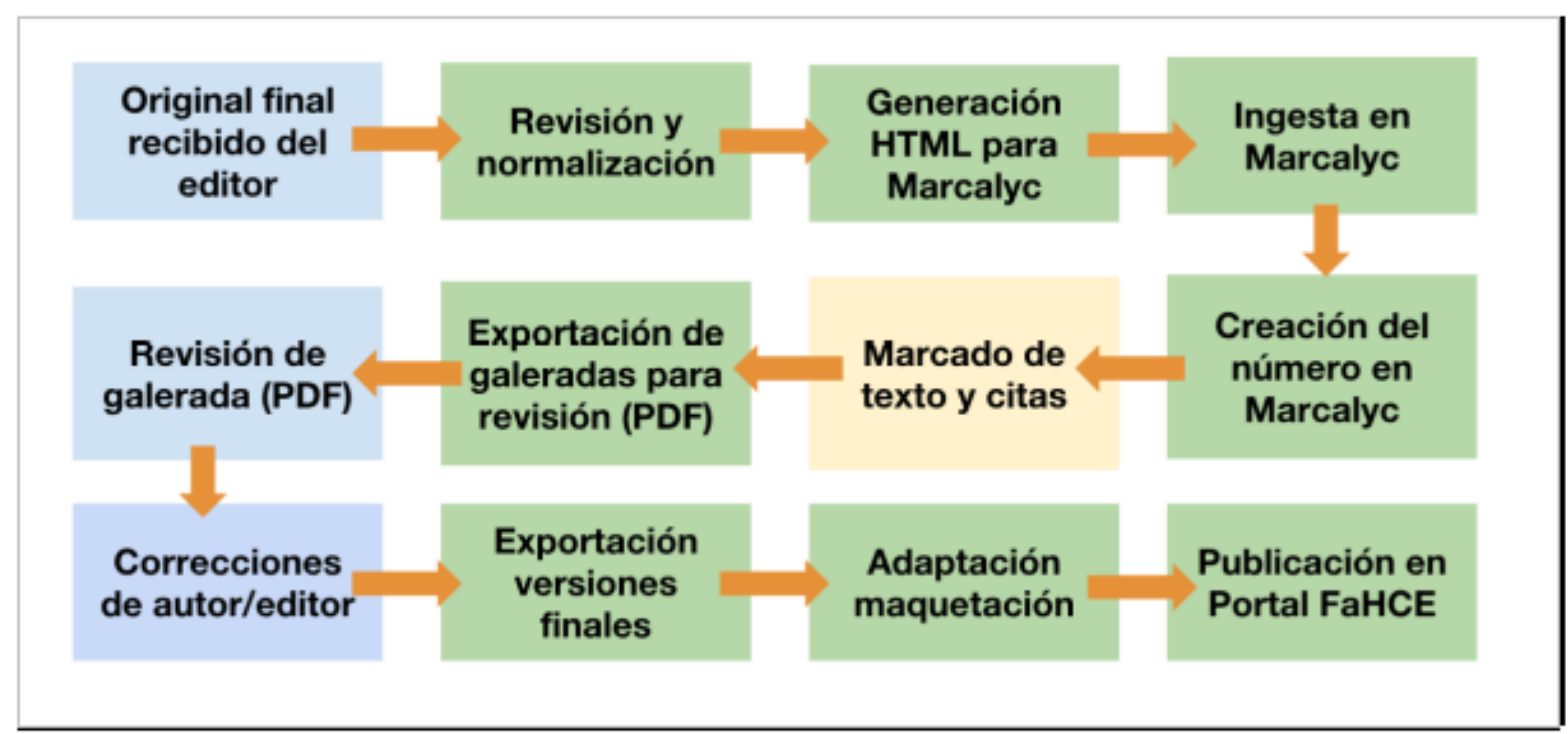

\footnotetext{
Elaboración propia

Referencias: Celeste: tarea realizadas por editores; Verde: tareas realizadas por profesionales del equipo técnico de edición; Amarillo: bibliotecarios externos al equipo técnico.
}

En el caso de la FaHCE-UNLP, este flujo se realiza de igual manera sobre las 16 revistas, con un aproximado de 360 contribuciones anuales. El equipo técnico está compuesto por 5 personas, un informático, dos bibliotecarios que controlan y trabajan sobre la maquetación final de cada trabajo (adaptación maquetación), un bibliotecario que controla que esta edición esté en relación a los metadatos en OJS y su futura indización, y una dirección también a cargo de un bibliotecario.

Es importante aclarar que con la única ingesta en HTML en la herramienta de marcado Marcalyc/xml, se producen al final del flujo cuatro formatos de salida (versiones finales): HTML, PDF, E-PUB y Visor, además de un xml-jats que se archiva como respaldo de cada ítem procesado. Sobre estas versiones finales, los bibliotecarios realizan modificaciones mínimas ${ }^{10}$ que hacen a la identidad de cada revista y la institución, pero bien podrían utilizarse sin modificaciones para la publicación directamente en OJS.

Una vez publicado el número completo se procede a su indización en diferentes portales: RedALyC, SciELO (en adaptación), DOAJ, Google Scholar, Repositorio institucional, etc.

\section{ESTIMACIÓN DE COSTOS EN RECURSOS HUMANOS}

Hemos realizado una estimación sobre los costos en recursos humanos que implica llevar adelante este flujo de trabajo. Entendemos que un análisis de costos más afinado debería contemplar los bienes de uso, tanto como los servicios de luz, gas y teléfono en la oficina, pero como éstos quedan subsumidos en una institución que se compone de cinco edificios y utiliza más de mil computadoras, las seis que componen nuestro inventario, con sus correspondientes escritorios (dos de ellos de más de veinte años), realmente puede decirse que no representan valores comparables a la inversión en trabajo profesional.

La Facultad de Humanidades y Ciencias de la Educación junto al Instituto de Investigaciones en Humanidades y Ciencias Sociales de doble dependencia UNLP-CONICET son los responsables editoriales de las 16 revistas científicas. Algunas, pocas, son anuales, la mayoría semestrales y dos cuatrimestrales, las cuales en total publican 35 números al año compuestos por alrededor de 360 artículos. 
Una vez que los editores han finalizado el proceso de evaluación, el procesamiento de los documentos es realizado por 5 personas que tienen una relación de dependencia muy variada, ya que el equipo se compone de una profesional de apoyo de CONICET, dos personal de apoyo académico y dos contratados que suman un total de 28 horas de trabajo por día. Estas se distribuyen de la siguiente manera:

- $4+4$ h: maquetación y metadatos en OJS3

- $8 \mathrm{~h}$ : indización y metadatos en OJS3

- 6 h: gestión

- 6 h: soporte técnico

La FaHCE-UNLP y el CONICET invierten en sueldos de este personal 2 contratos por $\$ 240.000$ anuales y 3 cargos de planta UNLP-CONICET por $\$ 1.560 .000$, haciendo un total de $\$ 1.800 .000$. A esto hay que sumarle el marcado con los sistemas Marcalyc/AmeliCA de los 360 artículos a un costo de $\$ 400$ cada uno, es decir \$144.000. En total, \$1.944.000 (U\$D 48.600). ${ }^{11}$ Por lo tanto, cada artículo cuesta \$ 5.400 (U\$D 135). De este modo, si lo comparamos con nuestra estimación de 2014 (\$8.331,7), hemos logrado reducir el monto por artículo, a partir de un modelo de gestión y un soporte tecnológico más eficiente.

Si extrapoláramos este modelo de trabajo a las 233 revistas que componen el Núcleo Básico de Revistas Científicas Argentinas, el país debería invertir: 22 artículos (promedio FaHCE)*233 revistas NBRCA*U \$D135 (por artículo) = U\$D 692.010. Esta suma representa el 3\% de lo que invierte la Biblioteca Electrónica de Ciencia y Tecnología (BECYT) para comprar los paquetes de títulos de las bases de datos comerciales cifra que, en 2016, ascendió a U\$D 21.998.510. ${ }^{12}$

Si por otra parte fuésemos más ambiciosos y quisiéramos realizar este trabajo de calidad sobre las revistas evaluadas para Catálogo Latindex, que antes de su reevaluación actual contaba con 742 revistas argentinas nos daría: 22 artículos (promedio FaHCE)*742 revistas Catálogo Latindex*U\$D 135 = U\$D 2.203.740, equivalente al $10 \%$ de la BECYT.

\section{ConCluSión}

Los desafíos para los editores latinoamericanos desde los niveles locales al contexto internacional han llevado a un proceso de profesionalización y de redefinición del Acceso Abierto. Quienes entendemos que el conocimiento es un bien común que debe permanecer financiado por fondos públicos, defendemos al mismo tiempo una definición del Acceso Abierto en la que los cargos al autor por procesamiento de los documentos no tiene lugar. Asimismo, consideramos que la soberanía del conocimiento es el fundamento que nos permite proteger el patrimonio cultural ancestral y científico.

$\mathrm{Al}$ mismo tiempo, estos fundamentos de gestión requieren herramientas tecnológicas que nos permitan competir con los modelos comerciales. Con los datos concretos expuestos en este trabajo podemos concluir que los costos para las instituciones de ciencia sostenidas por fondos públicos no son significativos en el total de sus gastos, y sí se pueden sostener los costos fijos de la edición científica, entonces ¿qué sentido tiene pagar por APC o suscripciones a editoriales comerciales?, tal como concluyen Larivière, Haustein y Mongeon (2015). Adicionalmente, tampoco hay gastos de venta ni de traslados, ya que los trabajos quedan disponibles directamente para el uso una vez finalizada la edición, con lo cual esos costos también son marginales para las editoras universitarias.

Por lo tanto, hemos demostrado que nuestro modelo de edición es sustentable, en una facultad perteneciente a una gran Universidad. Entonces, si el modelo de edición de revistas científico-académicas abierta es sustentable, el próximo paso es generar acciones para que los sistemas de evaluación consideren y apoyen el crecimiento de estas revistas como válidas, tanto como las comerciales y cambien en concordancia 
con la necesidad de hacer visible la producción editorial nacional y regional, ya que sin este último cambio todo nuestro esfuerzo habrá sido en vano.

En esta línea de cambios creemos que es necesaria una serie de acciones mancomunadas en los niveles nacional y supranacional que presione a los países a modificar los sistemas de evaluación para que todas las revistas sean valoradas con igual criterio, calidad científica y editorial, como así también ayude a impulsar políticas públicas para el financiamiento de la ciencia editada en la región, tales como, por ejemplo, tomar la decisión de invertir menos de un $10 \%$ de lo invertido en ver hacia afuera para ayudar a que se vea la ciencia editada en la región, tal como se mostró en el caso de Argentina: 20.000.000 U\$ para las bases de datos comerciales vs 2.000.000 U\$, que no se invierten, para optimizar la visibilidad de las producciones locales y regionales.

\section{REFerencias bibliográficas}

Aguado-López, E. (2018). La fragmentación del Acceso Abierto en Latinoamérica lo inserta en un futuro incierto [Mensaje de blog]. Blog Ameli. Recuperado de http://www.amelica.org/index.php/2018/11/07/la-fragmentac ion-del-acceso-abierto-en-latinoamerica-lo-inserta-en-un-futuro-incierto/

Aguado-López, E., y Vargas Arbeláez, J. E. (2016). Reapropiación del conocimiento y descolonización: el acceso abierto como proceso de acción política del sur. Revista Colombiana de Sociología, 39(2), 69-88. https://doi.org/10.15 446/rcs.v39n2.58966

Banzato, G. (2018). Soberanía del conocimiento para superar inequidades: políticas de Acceso Abierto para revistas científicas y repositorios institucionales en América Latina. Ponencia presentada en Maria Sibylla Merian Centre Conviviality-Inequality in Latin America International Workshop "Conviviality in Unequal Societies: The Knowledge Dimension", La Plata.

Becerril-García, A. (2019). AmeliCA vs Plan S: mismo objetivo, dos estrategias distintas para lograr el acceso abierto [Mensaje de blog]. Blog Ameli. Recuperado de http://www.amelica.org/index.php/2019/01/10/amelica-vs-pla n-s-mismo-objetivo-dos-estrategias-distintas-para-lograr-el-acceso-abierto/

Bosch, M. (2013). Revalorización y tangibilización de los recursos en información y edición científica en Argentina: líneas de trabajo y perspectivas del Centro de Información Científica y Tecnológica del CONICET. Salud Colectiva, 9(3), 281-285. Recuperado de http://www.redalyc.org/articulo.oa?id=73129417001

Caitán, N. (2011). Acceso al conocimiento científico-tecnológico: El rol de las políticas públicas. E-Colabora: Revista de Ciencia, Educación, Innovación y Cultura, 1(2). Recuperado de http://eprints.rclis.org/16157/

Claudio-González, M. G., Martín-Barranera, M., y Planas, A. V. (2017). La edición de revistas científicas en España: una aproximación descriptiva. Anales de Documentación, 20(1), 1-16. https://doi.org/10.6018/analesdoc.20.1 .265771

Declaración de Berlin. (2003, octubre 22). Recuperado de http://openaccess.mpg.de/67627/Berlin_sp.pdf

Declaración de Bethesda. (2003, abril 11). Recuperado de http://legacy.earlham.edu/ peters/fos/bethesda.htm

Declaración de Budapest. (2002, febrero 14). Recuperado de http://www.budapestopenaccessinitiative.org/translatio ns/spanish-translation

Larivière, V., Haustein, S., y Mongeon, P. (2015). The Oligopoly of Academic Publishers in the Digital Era. PLOS ONE, 10(6), e0127502. https://doi.org/10.1371/journal.pone.0127502

LATINDEX-REDALYC-CLACSO-IBICT (2017, diciembre 15). Declaración de México. A favor del Ecosistema Latinoamericano de Acceso Abierto No Comercial. Recuperado de http://www.accesoabiertoalyc.org/declaracio n-mexico/

Rafols, I., y Molas Gallart, J. (Trads.). (2015). El manifiesto de Leiden sobre indicadores de investigación. Recuperado de http://www.ingenio.upv.es/es/manifiesto

Repiso, R. (2014, mayo 6). A las puertas de Scielo (Web of Science) [Mensaje de blog]. EC3Metrics. Recuperado de h ttps://ec3metrics.com/las-puertas-de-scielo-web-science/ 
Rozemblum, C., y Banzato, G. (2012). La cooperación entre editores y bibliotecarios como estrategia institucional para la gestión de revistas científicas. Información, Cultura y Sociedad, 27. Recuperado de http://ppct.caicyt.go v.ar/index.php/ics/article/view/1983

Rozemblum, C., Bava, L., Unzurrunzaga, C., y Banzato, G. (2014). Costos y beneficios de la inclusión de revistas universitarias en bases de datos de Acceso Abierto. Trabajo presentado en 12a Jornada sobre la Biblioteca Digital Universitaria, Salta. Recuperado de http://www.memoria.fahce.unlp.edu.ar/trab_eventos/ev.4111/ev.4111.pd $\mathrm{f}$

The San Francisco Declaration on Research Assessment [DORA]. (2012). Recuperado de http://am.ascb.org/dora/

Vargas Arbeláez, J. E. (2014). Acceso abierto e instituciones de lo común: acción política en la academia latinoamericana. Crítica y Emancipación, VI(12), 357-400. Recuperado de http://clacsovirtual.org/pluginfile.p hp/39049/mod_resource/content/1/CyE12-4.pdf

\section{Notas}

1 Presentación invitada en el $3^{\circ}$ Congreso Internacional de Editores Redalyc "Construyendo el modelo de publicación académica del sur global”. Trujillo, Perú, 16-18 mayo 2018. http://congreso.redalyc.org/ocs/public/congresoEditores /index.html

2 Declaración de Berlín, 2003; Declaración de Bethesda, 2003; Declaración de Budapest, 2002.

3 Véase el artículo de Eduardo Aguado y Arianna Becerril en este dossier.

4 Rafols, I. y Molas Gallart, J. (Trads.) (2015). The San Francisco Declaration on Research Assessment (DORA), 2012

5 Al cambio oficial del 25/11/14 U\$D 977,8 https://www.lanacion.com.ar/1746786-dolar-oficial-dolar-blue-bolsa-por tena.

6 Traducción nuestra.

7 https://www.accesoabierto.net/dulcinea/, datos de 30/01/19.

8 "El concepto de soberanía alimentaria fue desarrollado por Vía Campesina y llevado al debate público con ocasión de la Cumbre Mundial de la Alimentación en 1996, y ofrece una alternativa a las políticas neoliberales" en https://viaca mpesina.org/es/que-es-la-soberania-alimentaria. Véase también la Declaración de Niélény de 2007: https://nyeleni.or g/spip.php?article291.

9 http://portal.amelica.org/ameli/

10 Éstas son: Inclusión de logos de la revista, licencias, citas sugeridas / DOI, estilos de acuerdo a identidad de cada revista (color en css de Marcalyc, alineaciones, espacios entre letras y párrafos, márgenes), paginación, y cambio de lugar de algunos elementos.

11 En un contexto de alta inflación y fluctuación de la moneda, estamos tomando un dólar a $\$ 40$.

12 http://www.biblioteca.mincyt.gob.ar/estadisticas/coleccion 